\title{
Forty Years of Clinimetrics
}

\author{
Giovanni A. Fava \\ Department of Psychiatry, University at Buffalo, State University of New York, Buffalo, NY, USA
}

In 1982, Alvan R. Feinstein introduced the term "clinimetrics" to indicate a domain concerned with indices, rating scales, and other expressions that are used to describe or measure symptoms, physical signs, and other distinctly clinical phenomena [1]. The customary taxonomy, in fact, did not include patterns of symptoms, severity of illness, effects of comorbid conditions, timing of phenomena, rate of progression of illness, functional capacity, and other clinical features that demarcate major prognostic and therapeutic differences among patients who otherwise seem deceptively similar because they have the same diagnosis and laboratory results [2]. He also added the psychosocial impact of ailment and treatment on individual, family and interpersonal relationships, including physical activities, joys, and sorrows of daily life [2]. In his original paper [1], Feinstein quoted as examples of clinimetric indices Jones criteria for rheumatic fever [1] and Apgar's method of scoring the newborn's condition [3]. The development of clinimetrics had the purpose to maintain and improve clinical art while advancing the state of clinical sciences [1].

In 1987, Feinstein published a monograph concerned with the rules that govern the structure of indices, the choice of component variables, the evaluation of consistency and validity [4]. The structure, choice of variables, and organization of output scales may greatly vary. The use that indices have in clinical medicine and psychiatry may range from diagnostic criteria of disease (determina-

karger@karger.com

(C) 2021 S. Karger AG, Basel

www.karger.com/pps

Karger! tion of status) to description of alterations recorded in repeated ratings (monitoring of change) and prognostic estimation (prediction) [4].

As with Feinstein's other scientific innovations [5], the concept of clinimetrics was far ahead of its time. It involved a paradigm switch [6] and was in sharp contrast with the prevailing climate. In particular, the large majority of instruments addressing psychosocial variables, such as quality of life and Patient-Reported Outcome Measures (PROMs), were using psychometric methods [7]. The clinimetric approach was often seen as redundant or unnecessary, without realizing that psychometric theory was an obstacle to the progress of clinical research $[8,9]$. Two major drawbacks could only be overcome by the clinimetric approach [9]. One is the psychometric assumption of the need for homogeneity of components of rating scales, as measured by statistical tests such as Cronbach's alpha and factorial analysis $[8,9]$. The redundant components that give a scale a high score for homogeneity are likely to obscure its capacity to record changes in clinical status [8-10]. In the clinimetric approach, neither homogeneity of components nor unidimensionality is required, in line with the heterogeneous features of clinical variables [8-10]. Further, in classical psychometrics, all items of a rating scale have the same weight and measure parallel forms of the same symptom [8-10]. Yet in clinical practice (as reflected by the clinimetric approach), not all symptoms have the same weight (major and minor symp- 
toms can be differentiated, such in Jones' criteria for rheumatic fever [1]). The misleading effects of the psychometric model become particularly evident using psychiatric diagnostic criteria [11]. Even though the DSM [12] is in line with the clinimetric model in terms of diagnostic criteria, the hidden conceptual model is psychometric: severity is determined by the number of symptoms, not by their intensity or quality, to the same extent that a score in a depression self-rating scale depends on the number of symptoms that are scored as positive [11]. This is not surprising in view of the fact that the development of psychometrics took place outside of the clinical field, mainly in educational and social areas $[8,9]$. Since the phenomena under observation in the development of psychometric principles were not clinical, they could not be automatically adapted to clinical psychology and psychiatry. For instance, a patient with severe and pervasive anhedonia, incapacitating fatigue and difficulties concentrating, which make him unable to work, would not be diagnosed as suffering from a major depressive disorder, whereas the diagnosis could be performed in a patient who barely meets the criteria for 5 symptoms [11]. This does not mean that everything developed by psychometric methods should be discarded. There are scales that were developed with psychometric methods and yet display excellent clinimetric properties, such as the Hamilton Rating Scales for Depression [13]. And there are psychometric constructs that find full room in the clinimetric approach, such as the concept of incremental validity (each distinct aspect of psychological measurement should deliver a unique increase in information in order to qualify for inclusion) [14].

The importance of clinimetrics, however, is not limited to the rules that shape and govern measurements in medicine. Clinimetrics paves the ground for setting a revolutionary approach to clinical medicine and psychiatry. Some features have been outlined in the latest review of the field that was published in 2012 [14]. However, clinimetric research that has been performed in the past decade has paved the way to additional clinical and research developments.

\section{The Inadequacy of the Disease Model and the Need of Broadening Clinical Focus}

There is increasing awareness of the inadequacy of disease as the primary focus of medical care. Tinetti and Fried [15] have observed that, when disease became the focus of medicine in the past two centuries, the average life expectation was 47 years, and most clinical encounters were for acute illness. The changed spectrum of health conditions (shifted toward aging and chronicity) and the interindividual variabilities in health priorities suggest that the aim of treatment should be the attainment of personal goals, upon identification of all modifiable biological and nonbiological factors involved [15]. Disease-specific guidelines provide very limited indicators for patients with multiple conditions [16]. Further, there are many symptoms and complaints that do not find adequate assessment in the medical system, may occur in up to $30-40 \%$ of medical patients and increase medical utilization costs [17]. Finally, in fields such as neurology and geriatrics, the main challenge is becoming to classify disability rather than disease [15, 16]. The challenge then becomes how to assess clinical variables, in addition to the disease definition, that may improve illness configuration, in terms of differential features and subtyping, course, and response to treatment [14]. Clinimetric research has greatly expanded the targets of clinical assessment in medicine and psychiatry. An illustration of some of the most representative areas where valid and reliable indices are available, is presented here.

\section{Allostatic Load}

A major limitation of current medical and psychiatric assessments is the lack of reference to psychosocial and environmental problems. Stressful life circumstances, occupational and financial problems, housing, family, and interpersonal frictions are examples. Allostatic load refers to the cumulative burden of chronic stress and life events $[18,19]$. It involves the activation and interaction of different physiological systems at varying degrees of activity. When environmental challenges exceed the individual ability to cope, then allostatic overload ensues [19]. The introduction of the concept of allostatic load sparked an increasing amount of research geared to the use of biological markers for its identification, as summarized in a recent systematic review [19]. The findings indicated that higher allostatic load was associated with poorer health outcomes in both general and clinical populations [19]. However, biomarkers express a state of body system that may be affected by a number of variables unrelated to stress and do not provide information on the underlying individual causes [19]. The introduction of clinimetric methods for assessment, such as a self-rated measurement of the level of allostatic load, the Psychosocial Index [20, 21 ], and diagnostic criteria for the determination of al- 
lostatic overload $[17,20]$, has greatly expanded the implications of the concept [19]. The joint use of biological and clinimetric criteria may greatly enhance the quality and precision of research in the field.

\section{Lifestyle}

An increasing body of evidence links the initiation and progression of several medical disorders, such as diabetes, obesity, and cardiovascular illness to lifestyle and behavior [22]. The benefits of modifying lifestyles were demonstrated in several randomized controlled trials [22]. Clinimetric tools for assessing lifestyle and related variables, such as alcohol consumption, nicotine dependence, and sleep quality, have become available [23].

\section{Psychological Well-Being and Euthymia}

An impressive body of investigations has suggested that positive affect and psychological well-being play a buffering role in coping with stress and have a favorable impact on disease course [17]. In recent years, there has been increasing interest in the concept of euthymia, a state characterized by positive affect, psychological flexibility, and resilience to stress [24]. Clinimetric tools for the determination of euthymia, such as the Clinical Interview for Euthymia and the Euthymia Scale, a self-rated inventory, have been developed [24]. Euthymia may play an important role in decreasing vulnerability to relapse, enhancing the level of recovery, and modulating mood in psychiatric disorders, as well as in improving coping and adaptation to medical disturbances [24].

\section{Illness Behavior}

Once the symptoms of a medical disease are perceived by a person, or he/she has been told by a doctor that he/she is ill even if symptoms are absent, then this disease-related information influences the patient's experience and behavior, as well as the course and therapeutic outcome, of a given illness episode [25]. The study of illness behavior (the ways in which individuals experience, perceive, evaluate, and respond to their own health status) has yielded important information in medical patients $[17,26]$. There are many manifestations of abnormal illness behavior, that range from hypochondriasis and disease phobia to illness denial and lack of compliance [26]. Illness behavior, because of its complexity, poorly fits psychometric limitations and needs a clinimetric assessment, such as the Diagnostic Criteria for Psychosomatic Research [17] or the Illness Attitude Scales [27].

\section{Organizing the Components of Clinical Assessment}

When Feinstein [28] introduced the concept of comorbidity, he referred to having disorders unrelated to the one of interest and to any "additional coexisting ailment" separate from the primary disease, even in the case this secondary phenomenon does not qualify as a disease per se. Indeed, in clinical medicine the many methods that are available for measuring comorbidity are not limited to disease entities [29]. Feinstein's conceptualization lends itself to various clinimetric strategies.

A unique feature of clinimetric tools is to provide a broad global rating of clinical phenomena $[2,3]$. One is concerned with a global evaluation of the overall severity of comorbidity by means of an index. The Charlson Comorbidity Index [30] is probably the most widely recognized method for assessing comorbidity as to the estimated risk of death $[31,32]$.

Another clinimetric method (staging) has achieved a major role in medical fields such as oncology and cardiovascular medicine $[2,14]$. Much later, it has been adopted in clinical psychiatry $[33,34]$. It may be based on the longitudinal development of disorders, their progression in terms of severity and complications, or their response to treatment.

A hierarchical organization of problematic areas (including focusing on the primary/secondary distinction) is purported by macro-analysis, a clinimetric functional method [10, 11, 14, 35]. It consists of the evaluation performed by the clinicians, using clinical and laboratory methods, of the various syndromes and problems that a patient may display, including psychosocial correlates, functional impairments, and treatment history. Many of the elements that can be used in macro-analysis are transdiagnostic, such as pain and illness behavior [14]. According to this model, a relationship between co-occurring syndromes and problems is established on the basis of where treatment should commence in the first place. Macro-analysis should be supplemented by micro-analysis, a detailed analysis of specific symptoms (onset and course of the complaints, circumstances that worsen symptoms and consequences) $[10,11,14,35]$.

\section{The Increasing Role of latrogenic Factors}

A rational use of drugs depends on the balance of potential benefits and adverse effects applied to the individual patient [36]. A problem in achieving such balance derives from the different sources of information that 
need to be integrated. Guidelines tend to place emphasis on systematic reviews and meta-analyses of randomized controlled trials that are uniquely geared to detecting benefits [37]. As a result, the prescribing clinician is driven by an overestimated consideration of potential benefits, paying little attention to the likelihood of responsiveness and to potential vulnerabilities in relation to the adverse effects of treatment [37]. Another major omission at a time of medication overload [38] is the lack of consideration of the role of iatrogenic components, particularly as related to drug treatment [39]. There have been major clinimetric developments in the assessment and definition of adverse events. Those that have occurred with psychotropic drugs, subsumed under the rubric of clinical pharmacopsychology [40, 41], stand as particularly representative. Clinimetric indices and a staging system for assessing subtle forms of behavioral toxicity, such as withdrawal phenomena and paradoxical effects, have been developed [39-42]. The concept of iatrogenic comorbidity refers to the unfavorable modifications in the course, characteristics, and responsiveness to treatment of an illness that may be related to previously administered therapies [39]. Such vulnerabilities may manifest themselves during treatment administration and/or after its discontinuation. Further, clinimetric research on medication attitudes has shed some new light on the phenomena related to adherence [43]. Finally, indices for measuring medication appropriateness have been validated [44]. These research efforts have expanded the assessment of iatrogenesis from the mere presence of new disorders to the detection of subtle and yet clinically meaningful manifestations.

\section{Identifying Therapeutic Components}

Placebo effects are often attributed to clinical interactions and contextual factors that affect expectations of the patient about the treatment and result in symptom changes [45]. The prevailing conceptualization consists of an undifferentiated placebo response that needs to be minimized in controlled investigations and maximized in clinical practice $[46,47]$. However, treatment outcome is the cumulative result of the interaction of several classes of variables with a selected treatment: living conditions (housing, nutrition, work environment, social support), patient characteristics (age, sex, genetic, general health conditions, personality, well-being), illness features and previous therapeutic experience, self-management, treatment setting (physician's attitude and atten- tion), and illness behavior [45]. Such variables may be therapeutic or counter-therapeutic. In certain patients, their interactive combination may lead to clinical improvement, whereas in other cases it produces no effect and, in a third group, it may lead to worsening of the condition [37]. Clinimetrics has provided the opportunity to measure these variables $[2,7,14,35]$. However, such measurements are generally omitted in placebocontrolled clinical trials, that are still based on lumping all these variables in an undifferentiated placebo response, using the narrow focus of obsolete psychometric methods of assessment [37]. Clinimetric tools are essential for interpreting the data. For instance, Lewis et al. [48] recently performed a randomized placebo-designed study of discontinuation or maintenance of fixed-dosing antidepressants in general practice patients who felt well enough to stop them. In a 52-week follow-up, the discontinuation group had a significantly higher relapse rate (56\%) compared to the maintenance group (39\%). The antidepressants that were used (citalopram, fluoxetine, sertraline, and mirtazapine), however, are frequently associated with withdrawal and post-withdrawal symptoms, that may be confounded with relapse [42, 49]. Without clinimetric criteria for their differential diagnosis $[42,50]$, there is no way to know how many of the relapses were actually withdrawal and post-withdrawal syndromes [51].

A comprehensive clinimetric approach to clinical trials may unravel synergistic actions between "specific" and allegedly "nonspecific" components. At the same time, in randomized clinical trials, lack of differences of medications, particularly psychotropic drugs, from placebo may be due to hidden counter-therapeutic ingredients.

\section{The Clinimetric Revolution in Research and Practice}

Clinimetric research in these 40 years has paved the ground for a long-awaited revolution in clinical research and practice, which encompasses three converging developments.

First, it may expand the current narrow biomedical model, that omits any reference to the psychosocial environment of the patient, to viewing illness as a result of interacting mechanisms at cellular, tissue, organismic, interpersonal, and environmental level, in line with Engel's biopsychosocial model [52] and McEwen's social neuroscience [53]. It is also in line with the concept of "exposome," as the totality of external conditions in our lives, including our physical and social environments, 
what we do in making choices, and how these choices affect our health-promoting and health-damaging behaviors [54].

Second, there is growing awareness that there is no simple "average" solution to most medical problems [37]. The question is how to put the available evidence within the context of individual, unique assets and liabilities. There is thus the need of integrating the information that derives from EBM with medicine-based evidence (MBE) [55]. MBE attempts to produce fine-grained profiling of subgroups of patients based on clinimetric criteria. The clinical advantages of this approach have recently been illustrated in clinical psychology and psychiatry [56], with particular reference to the coexistence of anxiety and depression [57].

Finally, clinical judgment remains the only viable option for selecting the main evidence-based treatment ingredients $[2,11]$. Its scientific use requires the information provided by clinimetric methods. As Horwitz et al. remarked, "what is needed to complement the power of genomics is an emphasis on personal attributes of patients and their environments, and to incorporate these features into an enriched approach to personalized medicine" [58, p. 1156].

This journal is pursuing clinimetric pathways. Like a laboratory, we are trying to improve clinical assessment and to remove the conceptual obstacles to the progress of clinical practice and research. In 2019, the journal has increased again its impact factor (IF) (17.66) and continues its growth. The new IF places Psychotherapy and Psychosomatics as fourth in the Science Citation Index Psychology ranking and fifth in the Science Citation Index Psychiatry ranking. The Appendix acknowledges the clinimetric work behind such achievement.

\section{Conflict of Interest Statement}

None

\section{Funding Sources}

The author has no funding to declare.

\section{Author Contributions}

The author conceived and wrote the entire manuscript.

\section{Appendix}

We would like to acknowledge here the work of the editorial board and statistical consultants; the help of many external reviewers (listed below), who dedicated their time and efforts to assess and improve the quality of submitted manuscripts; the skills of Emanuela Offidani and Andrea Sabbatini who prepared the press releases of the published articles; the support of our authors and readers. The following experts have supplemented the editorial board by reviewing the manuscripts submitted to Psychotherapy and Psychosomatics during 2021 and are gratefully acknowledged. Both external referees and editorial board members have disclosed potential conflicts of interests. The Editor-in-Chief and the Associate Editors have no conflicts of interest to declare for 2021.

M. Allen (Aarhus, Denmark)

A. Amad (Lille, France)

R. Baldessarini (Belmont, MA, USA)

L. Bartova (Vienna, Austria)

H. Baumeister (Ulm, Germany)

G. Belleville (Montreal, QC, Canada)

G. Benasi (New York, NY, USA)

E. Bernstein (Boston, MA, USA)

S. Blackwell (Bochum, Germany)

A. Buchheim (Innsbruck, Austria)

W. Carpenter (Baltimore, MD, USA)

D. Carrozzino (Bologna, Italy)

B. Cohen (Belmont, CA, USA)

R. Colle (Le Kremlin Bicêtre, France)

J. de Figueiredo (Cheshire, CT, USA)

A. Dingemans (Leiden, Netherlands)

N. Dittmer (Prien am Chiemsee, Germany)

M. Freeston (Newcastle, UK)

J. Favrod (Lausanne, Switzerland)

S. Gaigg (London, UK)

F. Galli (Rome, Italy)

C. Gastaldon (Verona, Italy)

D. Gorelick (Baltimore, MD, USA)

E. Granholm (Toronto, ON, Canada)

A. Gumley (Glasgow, UK)

J. Hamblen (Washington, DC, USA)

T. Heidenreich (Esslingen, Germany)

R. Holt (London, UK)

T. Joiner (Austin, TX, USA)

S. Kim (Bronx, NY, USA)

A. Karlamanglu (Los Angeles, CA, USA)

E. Karyotaki (Boston, MA, USA)

J. Kaufman (Montreal, QC, Canada)

C. Kellner (Charleston, SC, USA)

J. Klein (Lübeck, Germany)

E. Koster (Gent, Belgium)

J. Lancee (Amsterdam, Netherlands)

M. Linden (Berlin, Germany)

M. Lucente (Bologna, Italy)

G. Mansueto (Florence, Italy)

J. Margraf (Bochum, Germany)

D. Mataix-Cols (Stockholm, Sweden)

P. Meyer-Kalos (Minneapolis, MN, USA)

Y. Milaneschi (Amsterdam, Netherlands)

D. Moerman (Dearborn, MI, USA)

M. Mum-Chernoff (Chapel Hill, N.C. USA) 
A. Nardi (Rio de Janeiro, Brazil)

F. Neuner (Bielefeld, Germany)

J. Ogrodniczuk (Vancouver, BC, Canada)

S. Pallanti (Florence, Italy)

M. Pasquini (Rome, Italy)

C. Patierno (Bologna, Italy)

W. Pigeon (Rochester, NY, USA)

S. Priebe (London, UK)

M. Purgato (Verona, Italy)

M. Rimondini (Verona, Italy)

S. Schäfer (Greifswald, Germany)

M. Schiele (Freiburg, Germany)

N. Sonino (Padua, Italy)

V. Spormaker (Munich, Germany)

G. Taylor (Toronto, ON, Canada)

B. Thombs (Montreal, QC, Canada)

L. Tibi (Beer-Sheva, Israel)

\section{References}

1 Feinstein AR. T. Duckett Jones memorial lecture. The Jones criteria and the challenge of clinimetrics. Circulation. 1982 Jul;66(1):1-5.

2 Feinstein AR. An additional science for clinical medicine: IV. The development of clinimetrics. Ann Intern Med. 1983 Dec;99(6): 843-8.

3 Apgar V. A proposal for a new method of evaluation of the newborn infant. Curr Res Anesth Analg. 1953 Jul-Aug;32(1):260-7.

4 Feinstein AR. Clinimetrics. New Haven: Yale University Press; 1987.

5 Nierenberg AA, Sonino N. From clinical observations to clinimetrics: a tribute to Alvan FR. iesnteinFeinstein, MD. Psychother Psychosom. 2004;73:131-3.

6 Kuhn TS. The Structure of Scientific Revolutions. 2nd ed. Chicago: Chicago University Press; 1972

7 Carrozzino D, Patierno C, Guidi J, Berrocal Montiel C, Cao J, Charlson ME, et al. Clinimetric criteria for patient-reported outcome measures. Psychother Psychosom. 2021 Jul; 90(4):222-32.

8 Wright JG, Feinstein AR. A comparative contrast of clinimetric and psychometric methods for constructing indexes and rating scales. J Clin Epidemiol. 1992 Nov;45(11):1201-18.

9 Fava GA, Ruini C, Rafanelli C. Psychometric theory is an obstacle to the progress of clinical research. Psychother Psychosom. 2004 MayJun;73(3):145-8

10 Fava GA, Carrozzino D, Lindberg L, Tomba E. The clinimetric approach to psychological assessment: a tribute to Per Bech, MD (19422018). Psychother Psychosom. 2018;87(6): 321-6.

11 Fava GA, Rafanelli C, Tomba E. The clinical process in psychiatry: a clinimetric approach. J Clin Psychiatry. 2012 Feb;73(2):177-84.

12 Diagnostic and Statistical Manual of Mental Disorders: Fifth Edition. DSM-5. Washington, DC: American Psychiatric Association; 2013.
L. Tondo (Cagliari, Italy)

P. Tyrer (London, UK)

B. van Passel (Lent, Netherlands)

A. van Straten (Amsterdam, Netherlands)

C. Vazquez (Madrid, Spain)

R. von Känel (Zurich, Switzerland)

J. Volkert (Heidelberg, Germany)

T. Wahlund (Solna, Sweden)

D. Wallace (Kansas City, MO, USA)

S. Walther (Bern, Switzerland)

J. Wang (Shenzhen, China)

H. Ward (Boston, MA, USA)

K. Weimer (Ulm, Germany)

S. Wilhelm (Boston, MA, USA)

A. Wozniewicz (Torun, Poland)

R. Yu (Hong Kong, China)
13 Carrozzino D, Patierno C, Fava GA, Guidi J. The Hamilton rating scales for depression: a critical review of clinimetric properties of different versions. Psychother Psychosom. 2020; 89(3):133-50.

14 Fava GA, Tomba E, Sonino N. Clinimetrics: the science of clinical measurements. Int J Clin Pract. 2012 Jan;66(1):11-5.

15 Tinetti ME, Fried T. The end of the disease era. Am J Med. 2004 Feb;116(3):179-85.

16 Tinetti ME, Bogardus ST, Agostini JV. Potential pitfalls of disease-specific guidelines for patients with multiple conditions. $\mathrm{N}$ Engl J Med. 2004 Dec;351(27):2870-4.

17 Fava GA, Cosci F, Sonino N. Current psychosomatic practice. Psychother Psychosom. 2017;86(1):13-30.

18 McEwen BS. Protective and damaging effects of stress mediators. N Engl J Med. 1998; 338(3):171-9.

19 Guidi J, Lucente M, Sonino N, Fava GA. Allostatic load and its impact on health: a systematic review. Psychother Psychosom. 2021; 90(1):11-27.

20 Sonino N, Fava GA. A simple instrument for assessing stress in clinical practice. Postgrad Med J. 1998 Jul;74(873):408-10.

21 Piolanti A, Offidani E, Guidi J, Gostoli S, Fava GA, Sonino N, et al. Use of the PsychoSocial Index: a sensitive tool in research and practice. Psychother Psychosom. 2016 Oct;85(6): 337-45.

22 Rippe JM. Are we ready to practice lifestyle medicine? Am J Med. 2019 Jan;132(1):6-8.

23 Tomba E. Assessment of lifestyle in relation to health. Adv Psychosom Med. 2012;32:7296.

24 Fava GA, Guidi J. The pursuit of euthymia. World Psychiatry. 2020 Feb;19(1):40-50.

25 Lipowski ZJ. Physical illness and psychopathology. Int J Psychiat Med. 1974 Fall;5:48397.
26 Cosci F, Fava GA. The clinical inadequacy of the DSM-5 classification of somatic symptom and related disorders: an alternative trans-diagnostic model. CNS Spectr. 2016 Aug;21(4): 310-7.

27 Sirri L, Grandi S, Fava GA. The Illness Attitude Scales. A clinimetric index for assessing hypochondriacal fears and beliefs. Psychother Psychosom. 2008;77(6):337-50.

28 Feinstein AR. The pre-therapeutic classification of comorbidity in chronic disease. J Chronic Dis. 1970 Dec;23(7):455-68.

29 deGroot V, Beckerman H, Lankhorst GJ, Bouter LM. How to measure comorbidity: a critical review of available methods. J Clin Epidemiol. 2003 Mar;56:221-29.

30 Charlson ME, Pompei P, Ales KL, MacKenzie CR. A new method of classifying prognostic comorbidity in longitudinal studies: development and validation. J Chronic Dis. 1987; 40(5):373-83.

31 Charlson ME, Szatrowski TP, Peterson J, Gold J. Validation of a combined comorbidity index. J Clin Epidemiol. 1994 Nov;47(11): 1245-51.

32 Charlson ME, Wells MT, Ullman R, King F, Shmukler C. The Charlson comorbidity index can be used prospectively to identify patients who will incur high future costs. PLoS One. 2014 Dec;9(12):e112479.

33 Fava GA, Kellner R. Staging: a neglected dimension in psychiatric classification. Acta Psychiatr Scand. 1993 Apr;87(4):225-30.

34 Cosci F, Fava GA. Staging of mental disorders: systematic review. Psychother Psychosom. 2013;82(1):20-34.

35 Fava GA, Sonino N, Wise TN. The psychosomatic assessment. Basel: Karger; 2012.

36 Vanderbroucke JP, Psaty BM. Benefits and risks of drug treatments. How to combine the best evidence on benefits with the best data about adverse effects. JAMA. 2008 Nov;300: 2417-9. 
37 Fava GA. Evidence-based medicine was bound to fail: a report to Alvan Feinstein. J Clin Epidemiol. 2017 Apr;84:3-7.

38 Abramson J. Overdosed America. The Broken Promise of American Medicine. New Yok: Harper Perennial; 2004.

39 Fava GA, Rafanelli C. Iatrogenic factors in psychopathology. Psychother Psychosom. 2019;88(3):129-40.

40 Fava GA, Tomba E, Bech P. Clinical pharmacopsychology: conceptual foundations and emerging tasks. Psychother Psychosom. 2017; 86(3):134-40.

41 Cosci F, Guidi J, Tomba E, Fava GA. The emerging role of Clinical Pharmacopsychology. Clin Psychol in Europe. 2019;1(2):32158.

42 Fava GA. Discontinuing antidepressant medications. Oxford: Oxford University Press; 2021.

43 De Las Cuevas C, de Leon J. Reviving research on medication attitudes for improving pharmacotherapy. Psychother Psychosom. 2017 86(2):73-9.

44 Hanlon JT, Schmader KE. The Medication Appropriateness Index at 20: where it started, where it has been, and where it may be going. Drugs Aging. 2013 Nov;30:893-900.
45 Fava GA, Guidi J, Rafanelli C, Rickels K. The clinical inadequacy of the placebo model and the development of an alternative conceptual framework. Psychother Psychosom. 2017; 86(6):332-40.

46 Schedlowski M, Enck P, Rief W, Bingel U. Neuro-bio-behavioral mechanisms of placebo and nocebo responses. Pharmacol Rev. 2015 Jul;67:697-730.

47 Enck P, Bingel U, Schdlowski M, Rief W. The placebo response in medicine: minimize, maximize or personalize? Nature Rev. 2013 Mar;12:191-204.

48 Lewis G, Marston L, Duffy L, Freemantle N, Gilbody S, Hunter R, et al. Maintenance or discontinuation of antidepressants in primary care. N Engl J Med. 2021 Sep;385:1257-67.

49 Cosci F, Chouinard G. Acute and persistent withdrawal syndromes following discontinuation of psychotropic medications. Psychother Psychosom. 2020;89:283-306.

50 Chouinard G, Chouinard VA. New classification of selective serotonin reuptake inhibitor withdrawal. Psychother Psychosom. 2015;84: 63-71.
51 Fava GA. Are the same medications that are used in the acute treatment of depression the most suitable for preventing relapse? J Clin Psychopharmacol. 2021;41(Nov/Dec):617-9.

52 Fava GA, Sonino N. From the lesson of George Engel to current knowledge: the biopsychosocial model 40 years later. Psychother Psychosom. 2017;86(5):257-9.

53 McEwen BS. Epigenetic interactions and the brain-body communication. Psychother Psychosom. 2017;86(1):1-4.

54 Miller GW, Jones DP. The nature of nurture: refining the definitions of exposome. Toxicol. Sci. 2014 Jan;137:1-2.

55 Lobitz G, Armstrong K, Concato J, Singer BH, Horwitz RI. The biological and biographical basis of precision medicine. Psychother Psychosom. 2019;88(6):333-40.

56 Cosci F. Clinimetric perspectives in in clinical psychology and psychiatry. Psychother Psychosom. 2021;90(5):217-21.

57 Cosci F, Fava GA. When anxiety and depression coexist: the role of differential diagnosis using clinimetric criteria. Psychother Psychosom. 2021;90(5):308-17.

58 Horwitz RI, Cullen MR, Abell J, Christian JB. (De)personalized medicine. Science. 2012 Mar;339:1155-6. 